\title{
Clinical Audit on Current Practice of Anesthesia Machine Check and Equipment Preparedness Before Use in Debre Berhan Comprehensive Specialized Hospital, North Shewa, Ethiopia, 2020
}

\author{
Samuel Debas Bayable (D) \\ Melaku Bantie Fetene \\ Molla Amsalu Tadesse \\ College of Medicine and Health Science, \\ Debre Berhan University, Debre Berhan, \\ Ethiopia
}

\begin{abstract}
Background: Assessing the quality assurance of providing work-ready anesthesia equipment in operating rooms is so vital. Most of the anesthesia-related adverse events are preventable by the identification and correction of anesthesia machine errors and equipment preparation in the pre-induction period. This paper aimed to keep a high-quality environment for workers and patients in the surgical operating theater.

Materials and Methods: Data were collected after ethical clearance obtained from the institutional ethical review board, and daily observations of pre-induction anesthesia preparation were done in all surgical rooms at the start of each day and continuously before the next cases with a prepared anesthesia machine checklist. Descriptive statistical analysis and crosstabulation of each checklist with the type of anesthesia and urgency of surgery were done to determine the degree of staff compliance with daily preparations using SPSS version 22, and finally, results were presented by texts and graphs.

Results: In this study, a total of 61 checking events with 19 items were involved during the study period. There were $893(77.1 \%)$ fully met checking events, 205 (17.7\%) not-met checking events(sodalime) $61(5.3 \%)$ not available in anesthesia machine check and equipment preparation before use. Among 19 checklists, only three (power source plugged in, power source switched on, and functionality of monitoring) were perfectly completed; however, the rest sixteen checklists are in bad practice among them seven checklists (presence of functional self-inflating bag, automatic machine check, power source backup battery, anti-hypoxic device, ventilator, difficult airway equipment and scavenging systems) were checked below $90 \%$.
\end{abstract}

Conclusions and Recommendations: This study concludes there is poor compliance among anesthetists in pre-induction preparation and underperformed since all anesthesia machine checklists have zero tolerance for errors. . This paper recommends that every responsible anesthetist perform anesthesia machine check and equipment preparation regardless of the type of anesthesia and type of surgery and avoid professional negligence.

Keywords: anesthesia equipment preparedness, anesthesia machine, Audit; checklist

\section{Background}

Anesthesia equipment is vital to conduct safe anesthesia, therefor anesthetists are responsible to understand the function of the anesthetic equipment to ensure the correct function before use. ${ }^{1-4}$ Despite the growth in the number and quality of
Correspondence: Samuel Debas Bayable Tel +25I 9-II-43-75-07

Email samueldebas88@yahoo.com 
anesthesia providers, human error remains a major cause of anesthesia-related morbidity and mortality, which is accounts for up to $70 \%$ of anesthetic complications, ${ }^{5,6}$ among this insufficient anesthesia machine check has mostly associated with equipment-related morbidity. ${ }^{7,8}$

A study in Thailand on anesthetic equipment malfunction concludes ineffective equipment, lack of experience, ineffective monitors, and inadequate equipment are contributing factors, so to minimizing incidents equipment maintenance, pre-use equipment checking, vigilance, prior experience, compliance to guidelines and training are important. ${ }^{1,9}$

\section{Justification}

Anesthesia services are performed in diverse and complex settings which need per case preparation and checking of equipment before use. ${ }^{1,10}$ Anesthetists accomplish many tasks rapidly during induction of anesthesia, so the intense environment of the operating room, preparatory steps may be missed either unintentionally or possibly even intentionally to save time during emergency surgical procedures. $^{2}$

A Prospective study on anesthesia machine fault identification demonstrated that anesthesia machine check and equipment preparation before use continues to be a problem in the most operation room. ${ }^{11}$ Another study showed that among 200 surgical procedures twenty-three missed steps do occur at a significant and measurable rate that may subject patients to unnecessary risks. ${ }^{2,10}$

From clinical observation, full equipment check and preparation including anesthesia machine in-between cases has sometimes missed and a bundle of equipment problems has been reported from the operating theatres which is not actively repaired makes impressed to do this research.

The importance of this study is to assure and re-assure anesthesia machine check and equipment preparation before use to get $100 \%$ compliance and zero tolerance for error in anesthesia. ${ }^{3}$

\section{Methods}

A prospective observational study was done in 2020 at Debre Berhan comprehensive Specialized Hospital operation rooms, North Shewa, Ethiopia.

Professionals included in this study were six Master's, twenty BSc (Bachelor of Science), and two diplomas Anesthetists who served the hospital.

Data was collected after ethical approval was obtained from the Ethical Review Committee of the institute of medicine and Health Science College of Medicine, Debre Berhan University, using prepared anesthesia machine checklist consecutively by an assigned anesthetists during the study period. The study Performa was qualitative variables of yes or no and as necessary remark questions were filled from direct observation.

Descriptive Statistical analysis and cross-tabulation of each checklist with the type of anesthesia and urgency of surgery were done to determine the degree of staff compliance with daily preparations using SPSS (Statistical Package for Social Studies) version 22, and results were presented by texts and graphs.

\section{Results}

In this study, a total of 61 checking events with 19 items were involved during the study period. There were 893 (77.1\%) fully met checking events, 205 (17.7\%) not-met checking events $61(5.3 \%)$ not available in pre-induction preparation. Most surgical procedures (72.1\%) were emergency, and $62.3 \%$ of surgical procedures were done under regional anesthesia.

In this study, only three checklist manuals (power source plugged in, power source switched on, and functionality of monitoring) were perfectly completed, while correct gas outlet selection was done 60(98.4\%), oxygen flush,patency of breathing systm, filling of cylinder and airway equipment preparation were each performed 59(96.7\%) of the time.But availablity of functional suction, vaporizer (fitness,filled and leak free), and flow meter were checked 57()93.4\%,56 (91.8\%) and 55(90.2\%) respectively. however, the rest seven checklists are performed below $90 \%$ (presence of functional self-inflating bag 35(57.4\%), automatic machine check 19(31.1\%, power source backup battery41(67.2\%), anti-hypoxic device 30(49.2\%), ventilator 35(57.4\%), difficultairway equipment preparation $52(85.2 \%)$ ) and scavenging systems $27(44.3 \%)$ ) were checked.

Even though nearly all anesthesia machine checklist standards recommend anesthesia machine check and equipment preparation is the same for all types of anesthesia and all types of surgical procedures, a cross-tabulation results of each checklist with anesthesia preference and urgency of surgery in Figures 1 and Figure 2 shows pre-induction preparation was better in general anesthesia and elective surgical procedures, respectively.

\section{Discussion}

According to most standards of anesthesia machine check and equipment prepartions are routinely performed by the 


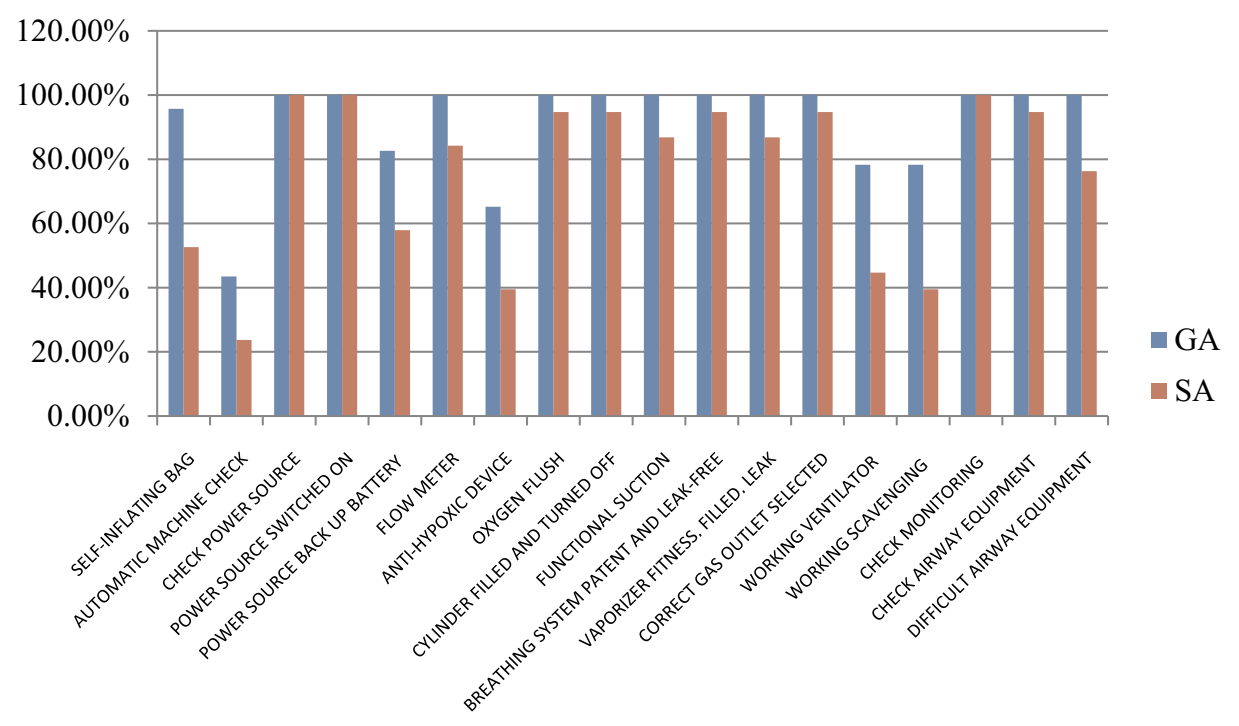

Figure I Cross-tabulation results showing the performance of anesthesia machine check and equipment preparedness before use with the type of anesthesia preference in Debre Berhan comprehensive Specialized Hospital, North Shewa, and Ethiopia 2020 (N=6I).

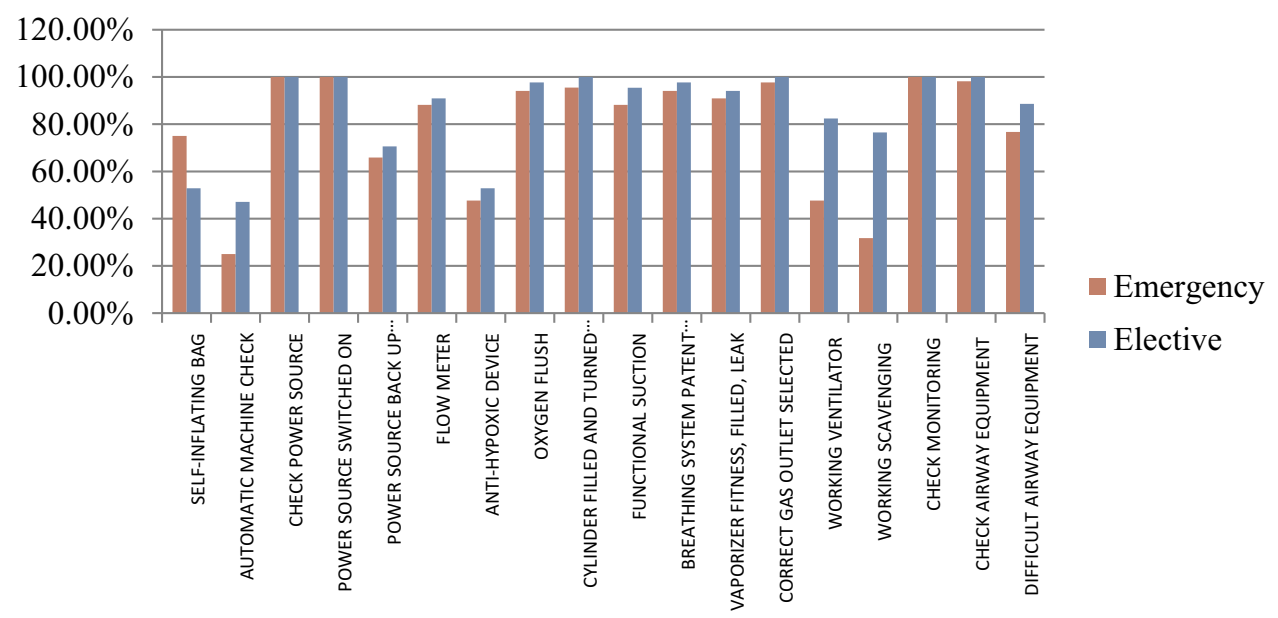

Figure 2 Cross-tabulation results showing the performance of anesthesia machine check and equipment preparedness before use with the urgency of the surgery in Debre Berhan comprehensive Specialized Hospital, North Shewa, Ethiopia 2020(N=61).

responsible anesthetist extensively in the morning and then with the fast track before each surgical case.

This study showed that the current practice of anesthesia machine check and equipment preparation is underperformed when compared to most anesthesia machine checklist recommendations, the possible reasons might be professional frustration to check events frequently due to COVID-19 pandemic, professional negligence, emergency surgeries preponderance $72.1 \%$ (anesthetists might rush to save time).

Even though most anesthesia machine checklist recommends anesthesia machine check and equipment preparedness are the same for all types of anesthesia and all types of surgical procedures, this study shows that pre-induction preparation was better in general anesthesia and elective surgical procedures.

Nowadays modern anesthesia is safe due to the discovery of predictable drugs but did not reduce dependence on anesthesia machines since the utilization of gas delivery, circuits, ventilator, and integrating monitoring devices that may induce risk related to machine failure., ${ }^{3,12}$ so checking the anesthetic equipment before daily use and between cases is an essential set up procedures. ${ }^{3,13}$ 
Studies conclude that $31 \%$ of equipment problems involved insufficient checking of the anesthesia machine, breathing circuit, and integrated monitoring before use, especially between cases., ${ }^{3,14}$ on the other hand, many studies clearly showed that safety issue and risky variables related to anesthesia machine check and equipment preparation before use is crucial. ${ }^{15-17}$

Most anesthesia-related adverse events are preventable by identification and correction of errors, so the checklist is mandatory even in a hectic and stressful clinical environment to reduce missing items in a standard induction protocol. ${ }^{1,18}$ Another study in Norway concludes that following regular inspection and a clear checklist of the manufacturers can contribute to abort any possibility of the hazard of anesthesia during operation, ${ }^{3,19}$ which is in line with this study.

\section{Conclusion and Recommendations}

This study concludes there is poor compliance among anesthetists in pre-induction preparation and underperformed since all anesthesia machine checklists have zero tolerance for errors.

This paper recommends that every responsible anesthetist should perform anesthesia machine check and equipment preparation regardless of the type of anesthesia and type of surgery and avoid professional negligence.

\section{Abbreviations}

SPSS, Statistical Package for Social Studies.

\section{Data Sharing Statement}

Data and materials used in this study are available and can be presented by the corresponding author upon reasonable request.

\section{Ethical Approval}

Ethical approval was obtained from the Ethical Review Committee of the institute of medicine and Health Science College of Medicine, Debre Berhan University and informed consent were waived by the ethics committee.

\section{Acknowledgments}

We would like to thank Debre Berhan University for giving us the chance to carry out this research. We also acknowledge the data collectors and staff of the Department of Anesthesia for their help and encouragement in conducting this study..

\section{Author Contributions}

All authors made a significant contribution to the work reported, whether that is in the conception, study design, execution, acquisition of data, analysis and interpretation, or in all these areas; took part in drafting, revising or critically reviewing the article; gave final approval of the version to be published; have agreed on the journal to which the article has been submitted; and agree to be accountable for all aspects of the work.

\section{Disclosure}

Samuel Debas Bayable reports a patent. Melaku Bantie Fetene reports non-financial support from Debre Berhan University during the conduct of the study and outside the submitted work. The authors report no other potential conflicts of interest for this work.

\section{References}

1. Hartle A, Anderson E, Bythell V, et al. checking anaesthetic equipment 2012: association of anaesthetists of Great Britain and Ireland. Anesthesia. 2012;67(6):660-668. doi:10.1111/j.1365-2044.2012.07163.x

2. Demaria S, Blasius K, Neustein SM. Missed steps in the anesthetic set-up. Anesth Analg. 2011;113(1):84-88. doi:10.1213/ ANE.0b013e318219645e

3. Al Suhaibani M, Al Malki A, Al Dosary S, Al Barmawi H, Pogoku M. Pre-use anesthesia machine check; certified anesthesia technician based quality improvement audit. Anesth Essays Res. 2014;8(3):354. doi:10.4103/0259-1162.143142

4. Lockey D, Crewdson K, Davies G, et al. AAGBI: safer pre-hospital anesthesia 2017: association of anaesthetists of Great Britain and Ireland. Anesthesia. 2017;72(3):379-390. doi:10.1111/anae.13779

5. Blike G, Biddle C. Pre anesthesia detection of equipment faults by anesthesia providers at an academic hospital: comparison of standard practice and a new electronic checklist. AANA J. 2000;68 (6):497-505.

6. Craig J, Wilson M. A survey of anesthetic misadventures. Anesthesia. 1981;36(10):933-936. doi:10.1111/j.1365-2044.1981.tb08650.x

7. Merry AF, Cooper JB, Soyannwo O, Wilson IH, Eichhorn JH. An iterative process of global quality improvement: the International Standards for a Safe Practice of Anesthesia 2010. Can J Anaesth. 2010;57(11):1021-1026. doi:10.1007/s12630-010-9380-7

8. Homra B, Clark A. Equipment Problems. Catastrophic Perioperative Complications and Management. Springer; 2019:305-316.

9. Kusumaphanyo C, Charuluxananan S, Sriramatr D, Pulnitiporn A, Sriraj W. The Thai anesthesia incident monitoring study (Thai AIMS) of anesthetic equipment failure/malfunction: an analysis of 1996 incident reports. $J$ Med Assoc Thailand = Chotmaihet Thangphaet . 2009;92(11):1442-1449.

10. Sweeney N, Owen H, Fronsko R, Hurlow E. An audit of level two and level three checks of anesthesia delivery systems performed at three hospitals in South Australia. Anesth Intensive Care. 2012;40 (6):1040-1045. doi:10.1177/0310057X1204000617

11. Larson ER, Nuttall GA, Ogren BD, et al. A prospective study on anesthesia machine fault identification. Anesth Analg. 2007;104 (1):154-156. doi:10.1213/01.ane.0000250225.96165.4b

12. Qadir N, Takrouri M, Seraj M, et al. Critical incident reports. Middle East J Anaesthesiol. 1998;14(6):425-432. 
13. Poepsel MSM. The Scope, Clinical Preparedness, and Contributing Factors to Rural Anesthesia Practice. Walden University; 2013.

14. Buffington CW, Ramanathan S, Turndorf H. Detection of anesthesia machine faults. Anesth Analg. 1984;63(1):79-82. doi:10.1213/ 00000539-198401000-00016

15. Amucheazi AO, Ajuzieogu O. Critical incidents during anesthesia in a developing country: A retrospective audit. Anesth Essays Res. 2010;4(2):64. doi:10.4103/0259-1162.73508

16. Enright A, Merry A. The WFSA and Patient Safety in the Perioperative Setting. Springer; 2009.

17. Turkistani A, Ael-Dawlatly A, Delvi B, Alotaibi W, Abdul Ghani B. Critical Incident Monitoring in a Teaching Hospital. Department of Anesthesiology American University of Beirut Medical Center PO Box 11-0236. Beirut 1107-2020, Lebanon. 2009;20(1):97.
18. Thomassen Ø, Brattebø G, Søfteland E, Lossius H, Heltne JK. The effect of a simple checklist on frequent pre-induction deficiencies. Acta Anaesthesiol Scand. 2010;54(10):1179-1184. doi:10.1111/ j.1399-6576.2010.02302.x

19. Fasting S, Gisvold S. Equipment problems during anesthesia-are they a quality problem? Br J Anaesth. 2002;89(6):825-831. doi:10.1093/bja/aef276

\section{Clinical Audit}

\section{Publish your work in this journal}

Clinical Audit is an international, peer-reviewed, open access journal focusing on the processes and outcomes of clinical audit in any area of healthcare. All aspects of patient care are addressed within the journal and practitioners from all disciplines are invited to submit their work. Areas covered include: Publication of audits; How an audit has changed practice; Practical tips on how to do audits and to

Submit your manuscript here: https://www.dovepress.com/clinical-audit-journal

\section{Dovepress}

avoid pitfalls; How audits have changed patient care; Calls and justifications for new audits. The manuscript management system is completely online and includes a very quick and fair peer-review system, which is all easy to use. Visit http://www.dovepress.com/ testimonials.php to read real quotes from published authors. 